\title{
Adaptación argentina de la guía de indicadores de calidad de vida para organizaciones que apoyan a personas con trastornos del espectro autista Argentine adaptation of the Quality of Life Indicators guide for organizations that support people with autistic spectrum disorders
}

\author{
Dr. José L. Cuesta Gómeza,b y Dra. Luisa A. Manzone $e^{b, c, d, e}$
}

\section{RESUMEN}

Este artículo describe el proceso de adaptación en Argentina de la guía de indicadores de calidad de vida para organizaciones que prestan apoyo a personas con trastornos del espectro autista (Cuesta, J. L., 2009), a través de la técnica Delphi, en la que han participado un grupo de expertos representativos de diferentes ámbitos e instituciones del país, relacionados con el autismo. El resultado es un instrumento, basado en el modelo de calidad de vida, adaptado al contexto argentino, que ayuda a planificar y evaluar centros y programas para personas con trastornos del espectro autista y da respuesta al creciente número de servicios específicos que responden a las necesidades de este colectivo. Palabras clave: trastorno del espectroautista, calidad de vida, organización y administración.

a. Facultad de Educación, Universidad de Burgos.

b. Asociación Española de Profesionales del Autismo.

c. Fundación Trastornos del Espectro Autista (TEA).

d. Centro Argentino Integral de Tratamiento Individualizado (CAITI).

e. Asociación Argentina de Profesionales del Espectro Autista

Correspondencia: Dra. Luisa A. Manzone: caitisrltea@gmail.com

Financiamiento: Ninguno.

Conflicto de intereses: Ninguno que declarar.

Recibido: 7-6-2017 Aceptado: 12-9-2017 http: / / dx.doi.org/10.5546/ aap.2018.e257

Texto completo en inglés:

http:/ / dx.doi.org/10.5546/ aap.2018.eng.e257

Cómo citar: Cuesta Gómez JL, Manzone LA. Adaptación argentina de la guía de indicadores de calidad de vida para organizaciones que apoyan a personas con trastornos del espectro autista. Arch Argent Pediatr 2018;116(2):e257-e266.

\section{INTRODUCCIÓN}

La búsqueda de la calidad de vida, actualmente, es un referente que guía la planificación y el desarrollo de las organizaciones de atención a personas con discapacidad. ${ }^{1}$ Las personas con trastorno del espectro del autismo (TEA) y sus familias requieren servicios (socioeducativos y sanitarios) que abarquen diferentes ámbitos y momentos del ciclo vital, y, dada la desigualdad en su desarrollo en los diferentes países, se debe contar con herramientas que permitan evaluar si estos reúnen las necesarias condiciones que garanticen su especificidad, especialización y aseguren la calidad de vida.
Al aproximar el concepto de calidad de vida a las entidades que integran a personas con TEA, se encuentra que este colectivo manifiesta graves dificultades comunicativas, en muchos casos, imposibilidad para expresar necesidades, deseos, nivel de satisfacción, estados físicos o emocionales, en definitiva, para compartir información sobre sí mismas, cuestiones básicas para evaluar su nivel de calidad de vida. ${ }^{2-12}$ Estas dificultades justifican la necesidad de contar con instrumentos que garanticen que las organizaciones y la intervención que se presta en sí mismas promuevan y garanticen el máximo nivel de calidad de vida.

Ante esta realidad, la investigación planteó que, sin excluir la utilización de escalas o instrumentos que evaluaran la dimensión subjetiva, percepción de las propias personas, cobraba una especial importancia profundizar en la dimensión objetiva como vía para poder evaluar y promover la calidad de vida. Dichas cuestiones, unidas al hecho de la dificultad de evaluar la calidad de vida en las personas con TEA desde una perspectiva subjetiva, hacen especialmente necesario validar indicadores que ayuden a favorecer determinadas condiciones en el entorno de la persona con TEA. ${ }^{13-15}$ El entorno o contexto de intervención debe, en sí mismo, potenciar el desarrollo personal y la máxima participación de las personas con TEA a lo largo de su vida, tal como plantea uno de los principales referentes en 
discapacidad, la Clasificación Internacional del Funcionamiento de la Discapacidad y de la Salud. ${ }^{13}$

Este trabajo surgió de la necesidad de disponer de una herramienta, adaptada al contexto argentino, que ayudara a planificar y evaluar en qué medida los centros y programas estaban mejorando la calidad de vida de las personas con TEA. La necesidad se justifica, además, por los resultados de diferentes estudios realizados en Argentina, que evidencian un aumento en la prevalencia del autismo, en la línea de otros estudios a nivel internacional: 4,5 personas por cada 1000 nacimientos; ${ }^{16} 4,8$ por cada $1000^{17} \mathrm{y}$ 14,6 por cada $1000 .{ }^{18}$

Tras una revisión de los instrumentos de evaluación de calidad de vida existentes, se comprobó que la mayoría no fueron diseñados para personas con TEA ${ }^{19-22} \mathrm{y}$, únicamente, se encontró un instrumento específico, la guía de indicadores de calidad de vida para organizaciones que prestan apoyo a personas con TEA ${ }^{23}$ Esta herramienta fue diseñada y validada en España, basándose en las contribuciones de los principales referentes en investigación sobre los TEA, ${ }^{24-26}$ en guías de buenas prácticas ${ }^{11,27}$ y a partir del consenso de grupo de 12 profesionales expertos en autismo, pertenecientes a diferentes servicios de apoyo de distintas organizaciones representativas del país, a través de la técnica Delphi. ${ }^{23}$ Con el objetivo de asegurar la máxima representatividad, el grupo de expertos tenían distintos perfiles profesionales y formación de base y una experiencia mínima de cinco años de trabajo con personas con TEA, con diferentes edades y niveles de funcionamiento.

La guía de indicadores de calidad constituye un instrumento de evaluación desde una perspectiva objetiva y contempla aquellos factores contextuales referidos a las organizaciones y servicios donde se integra a las personas con TEA que pueden incidir significativamente, de forma directa o indirecta, en su calidad de vida.

El instrumento consta de 67 indicadores agrupados en seis ámbitos: 1-calidad referida a la persona, 2-identificación de las necesidades, elaboración y seguimiento de los planes de desarrollo personal, 3- formación de profesionales, 4- estructura y organización, 5- recursos personales, materiales y espaciales y 6- relación con la comunidad/proyección social.

Cada indicador consta de cuatro evidencias, cuatro pruebas que ayudan a observar y hacer cuantificable el indicador y a poder asegurar si se cumple o no, con un mismo criterio de evaluación objetivo para todos los evaluadores.

El diseño de la guía permite su aplicación en diferentes centros y servicios de apoyo y contextos culturales, pues se centra en evaluar aspectos que se consideran comunes, independientemente de la edad de las personas con TEA o del tipo de servicio que reciban. Además, la evaluación se plantea de forma flexible y permite no aplicar algún indicador, si el grupo evaluador considera que no procede.

El objetivo general de la investigación que aborda este artículo es realizar la adaptación en Argentina de la guía de indicadores de calidad de vida para organizaciones que prestan apoyo a personas con TEA.

\section{MÉTODO}

\section{Selección de la técnica de investigación}

Para conseguir el objetivo de este trabajo, se ha utilizado la técnica Delphi, ${ }^{28,29}$ que es una técnica de consenso enmarcada en la metodología cualitativa, perteneciente al tipo de entrevista de grupo en profundidad.

La aplicación de esta herramienta busca lograr el consenso a través de la participación de un grupo de expertos especializados en distintos aspectos de un mismo campo para asegurar un análisis en profundidad desde distintas perspectivas y focalizar la atención a partir de diferentes planteamientos. ${ }^{30-32}$

Como ventajas de la utilización del correo electrónico, frente a la reunión física de grupos en la que se convoca a los expertos para que, en directo, realicen aportes e intercambien opiniones, se destacan el anonimato, la retroalimentación controlada, la independencia, la representatividad del grupo y la triangulación de datos e investigadores. . $8,29,33^{2}$

\section{Creación del equipo de expertos}

En este trabajo, ${ }^{28}$ el investigador asumió el rol de observador participante, un papel por el que se integró también en el grupo de participantes, y realizó aportes y propuestas que se sumaron al proceso de consenso de todo el grupo. ${ }^{28}$

La función del panel de expertos fue la de revisar la guía de indicadores, en distintas fases, con el objetivo de adaptar la terminología, asegurar la representatividad de los indicadores y evidencias, asegurar la objetividad de las evidencias y aportar cuantas sugerencias y propuestas consideraran necesarias para mejorarla.

La selección del grupo de expertos la realizaron los coordinadores del proyecto, 
autores del presente artículo, de acuerdo con los siguientes criterios:

- Trabajo en el campo de los TEA, con una experiencia superior a cinco años y reconocido prestigio.

- Representación de distintas disciplinas relacionadas con los TEA, que aportaran visiones complementarias.

- Representación de áreas geográficas diferentes. Teniendo en cuenta estos criterios, se estimó que el grupo de expertos se conformara con diez profesionales procedentes de universidades, centros educativos terapéuticos, centros de investigación y de atención a personas con TEA, escuelas de educación especial y de las siguientes localidades: Ciudad Autónoma de Buenos Aires (CABA), zona oeste de la Provincia de Buenos Aires, zona norte de la Provincia de Buenos Aires, Río Negro y Mendoza.

Los expertos representaban disciplinas complementarias relacionadas con el autismo: psicología, fonoaudiología, psiquiatría y educación. En el grupo, fueron incluidos expertos con reconocida experiencia en las distintas etapas evolutivas y en los campos de diagnóstico, intervención directa, apoyo a familias, salud e investigación.

\section{Instrumento de referencia}

Tal como se ha mencionado anteriormente, el instrumento de referencia para la investigación consta de 67 indicadores que, como medida de evaluación, son útiles para mejorar resultados. Su medida es significativa e interpretable y permiten la recogida de datos de forma sencilla. ${ }^{23}$

\section{Procedimiento}

El proceso de adaptación se inició en 2015, desde la Fundación TEA (Buenos Aires), con una fase exploratoria en la que, tras la formación del grupo de expertos, se procedió a preparar la $1^{a}$ versión de la guía de indicadores, que permitió comenzar la secuencia metodológica consistente en tres rondas o envíos por e-mail ${ }^{30}$ a través de los cuales se realizó una revisión cualitativa y se consensuaron las adaptaciones lingüísticas (véase el Anexo 1).

Los 10 expertos iniciales que se contactaron participaron activamente durante todo el proceso.

A partir de cada uno de los envíos, se analizaron las propuestas recibidas y se integraron aquellas con mayor nivel de consenso. Cada envío de información se inició con una respuesta individualizada a cada experto con información acerca de cómo se habían tenido en

TABLA 1. Ejemplo de adaptaciones terminológicas a partir de la versión española

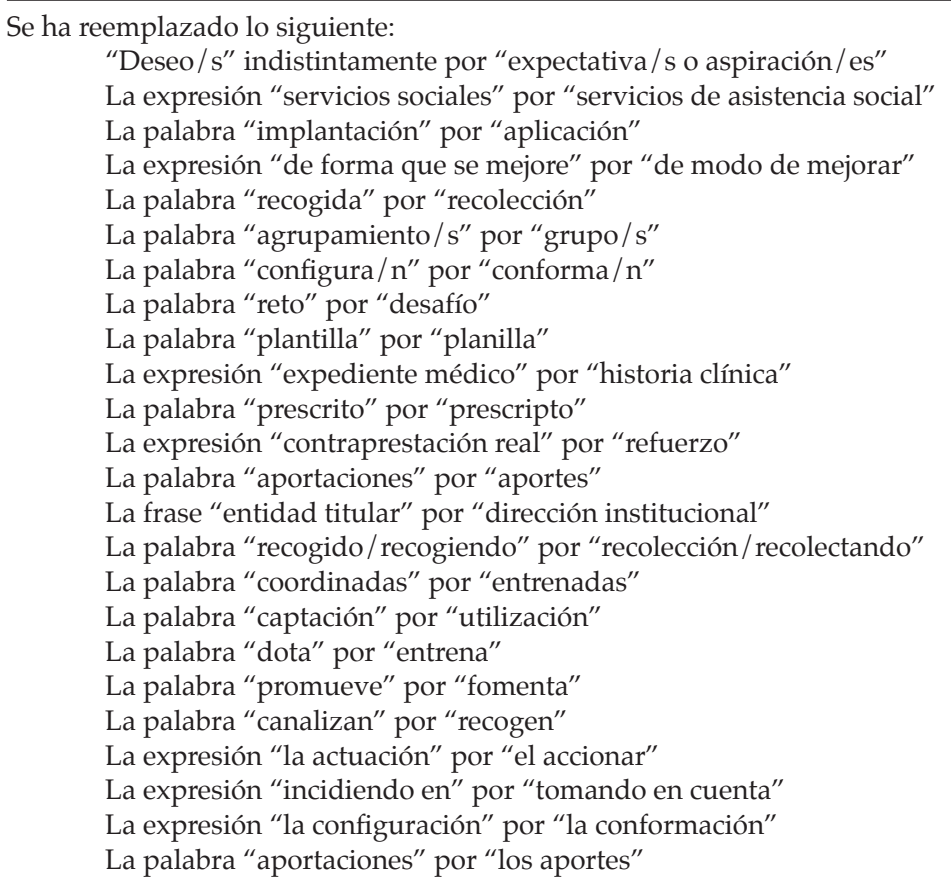


cuenta sus aportes y una información general a todo el grupo con aquellos aportes que habían tenido un mayor nivel de consenso.

\section{Primer envío}

El objetivo de esta ronda fue la revisión y la realización de los ajustes cualitativos que los expertos consideraron importantes, a partir de la $1^{\text {a }}$ versión de la guía de indicadores y su metodología de aplicación. Hubo un acuerdo de todo el grupo en relación con la definición y el modelo de calidad de vida que justificaba la estructura de la guía en la representatividad de los ámbitos, indicadores y evidencias, y acerca de la metodología de aplicación de la herramienta.

\section{Segundo envío}

La función del panel de expertos fue la de hacer la revisión terminológica de los contenidos de la guía de indicadores.

Los aportes más significativos permitieron matizar o sustituir algunas expresiones que dificultaban una correcta comprensión de los indicadores y evidencias. Además, se ajustó la denominación de términos referidos a perfiles profesionales que se mencionaban en la guía y se sustituyeron o aclararon algunos conceptos referidos a la intervención con las personas con TEA (véase la Tabla 1).

\section{Tercer envío}

En esta última fase, se sintetizaron todos los aportes obtenidos hasta el momento, y se realizó una consulta iterativa a los expertos sobre la versión definitiva de la guía y su metodología de aplicación. Al mismo tiempo, se preguntó a los expertos sobre la conveniencia de adaptar algunos conceptos planteados en el grupo, que no habían tenido un elevado nivel de consenso, pero que los coordinadores consideraron importantes para que fueran nuevamente evaluados.

\section{RESULTADO DEL PROCESO DE VALIDACIÓN}

La guía de indicadores de calidad de vida resultante (véase el Anexo 2) consta de 67 indicadores, pues no se ha modificado el número en relación con la versión original ni los ámbitos en los que están agrupados.

Esta guía se considera sensible a los apoyos y condiciones de las organizaciones (planificación y evaluación de los programas y servicios para personas con TEA) en relación con la persona, necesarios para mejorar su calidad de vida.

Cada indicador consta de cuatro evidencias, que aseguran su concreción y objetividad, independientemente del contexto o del evaluador (véase la Tabla 2).

Uno de los resultados de este trabajo es el manual de aplicación, que complementa la guía de indicadores de calidad de vida, y que establece que se aplique, de forma consensuada, por parte de un equipo consultor que asegure al máximo el tener en cuenta no solo las variables objetivas, sino también las subjetivas más relevantes que pueden influir sobre los contextos de participación de la persona. ${ }^{23}$

Una vez en la organización, el equipo evaluador, compuesto por un evaluador externo, responsable del Servicio, un familiar y, en casos en que sea posible, una persona con TEA, comprobará cada uno de los indicadores a través de las evidencias.

La guía cuenta con un programa informático, "OTEA", en el que se introducen los datos y, de forma automática, informa de la puntuación global mediante un gráfico que facilita la interpretación de la situación de la organización respecto a cada indicador. ${ }^{23}$

El proceso concluye con la elaboración y presentación de un informe final (por parte) que incluirá observaciones generales, indicaciones sobre qué aspectos deben tenerse en cuenta para

TABLA 2. Ejemplo: evidencias del indicador 9

Indicador 9: La persona con TEA y/o su tutor participan en la planificación, ejecución y evaluación de su plan de elaboración individual.

- Evidencias:

- Existen canales de participación y expresión de las personas con TEA y / o sus representantes en relación con el plan de desarrollo individual.

- Se proporcionan apoyos para que las personas puedan llevar a cabo elecciones y decisiones.

- Se analizan y tienen en cuenta las aspiraciones o expectativas, preferencias e intereses de las personas.

- Las actividades se adaptan y estructuran para garantizar el éxito en su realización de la forma más autónoma posible. 
mejorar el nivel de calidad, las pautas de mejora, así como una fecha de revisión si fuera necesario.

\section{DISCUSIÓN}

Este trabajo describe el proceso de adaptación y validación en Argentina de la guía de indicadores de calidad de vida para organizaciones que prestan apoyo a personas con trastornos del espectro.

Un error frecuente en la investigación sobre calidad de vida ${ }^{34}$ consiste en no tener claro el propósito de lo que se quiere evaluar y, por tanto, no utilizar los sistemas más adecuados. En el caso de esta investigación, al pretender evaluar los programas o servicios y su adecuación a las personas que los reciben, resulta acertado recurrir a indicadores objetivos.

Los modelos más actuales de evaluación de calidad de vida se basan en una visión multidimensional, que abarca todas las áreas, ámbitos y contextos de la vida de la persona, y contempla una perspectiva objetiva, referida a las condiciones medibles del entorno que, en sí mismas, ayudan a que la persona tenga una vida mejor. ${ }^{1,3,8,15}$

\section{CONCLUSIONES}

El grupo de expertos participante en la validación de la guía de indicadores de calidad de vida en Argentina la ha considerado como un instrumento válido en su contexto para evaluar el impacto que los servicios tienen en la vida de las personas con TEA que los reciben.

Esta herramienta se plantea como un recurso necesario para las numerosas instituciones que ofrecen servicios para personas con TEA en el país, como una referencia para aquellas organizaciones que deseen abordar el reto de implantar modelos basados en calidad de vida y como un estímulo para todos aquellos que asuman el desafío y la ilusión de avanzar hacia nuevas formas de enriquecer la calidad de los apoyos que reciben las personas con TEA.

Como fase que complementará el proceso de adaptación en Argentina de la guía, se plantea su difusión y aplicación en distintas organizaciones para asegurar su fiabilidad y consistencia, proyecto que, actualmente, está en fase de desarrollo.

\section{REFERENCIAS}

1. Reinders HS, Schalock RL. How organizations can enhance the quality of life of their clients and assess their results: the concept of QOL enhancement. Am Intellect Dev Disabil. 2014;119(4):291-302.
2. Billestedt E, Gillberg IC, Gillberg C. Aspects of quality of life in adults diagnosed with autism in childhood. Autism 2011;15(1):7-20.

3. Cuesta Gómez JL, Anuncibay R, González Bernal J, et al. A guide to indicators for the evaluation of specialist autism centres, based on the quality-of-life model. Int J Dev Disabil 2017:1-9. [Acceso: 20 de septiembre de 2017]. Disponible en: http: / / dx.doi.org/10.1080/20473869.2017.1361672.

4. Maxey D, Kezar A. Revealing Opportunities and Obstacles for Changing Non-Tenure-Track Faculty Practices: An Examination of Stakeholders' Awareness of Institutional Contradictions. J High Educ 2015;86(4):564-94.

5. Autism Speak. Educating for Excellence: Training Paraprofessionals in ASD Best Practices. 2013. [Acceso: 20 de septiembre de 2017]. Disponible en: https: / / www. autismspeaks.org/sites/default/files/the_autism_ project_final_report.pdf.

6. Witwer AN, Lecavalier L. Examining the validity of autism spectrum disorder subtypes. J Autism Dev Disord 2008;38(9):1611-24.

7. Povey C, Mills R, Gómez de la Cuesta G. Autism and ageing. Issues for the future. Midlife and Beyond 2011; 230-232.

8. Sheldick R, Neger E, Shipman D, et al. Quality of life of adolescents with autism spectrum disorders: concordance among adolescents self-reports, parents reports, and parents proxy reports. Qual Life Res 2012;21(1):53-7.

9. ShipmanD, ShelrickR, Perrin E. Quality of life in adolescents with autism spectrum disorders: Reliability and validity of self-reports. J Dev Behav Pediatr 2011;32(2):85-9.

10. Stuart- Hamilton S, Griffith G, Totsika V, et al. The circumstances and support needs of older people with autism. 2009. [Acceso:20 de septiembre de 2017]. Disponible en:http: / / gov.wales/docs / dhss/report/100622olderpeo plewithautismreporten.pdf.

11. Güemes I, Martín Arribas MC, Canal Bedia R, et al. Evaluación de la eficacia de las intervenciones psicoeducativas en los trastornos del espectro autista. Madrid: Ministerio de Ciencia e Innovación-Instituto de Salud Carlos III; 2009.

12. Palomo Seldas R. Autismo: teorías explicativas actuales. Madrid: Alianza; 2017.

13. Barthélémy C, Fuentes J, Howlin P, et al. Persons with Autism Spectrum Disorders. Identification, Understanding, Intervention. Bruselas: Autism Europe; 2008.

14. Plimley LA. A review of quality of life issues and people with autism spectrum disorders. Br J Learn Disabil 2007; 35(4):205-13.

15. Cuesta-GómezJL, Vidriales-Fernández R, Carvajal-Molina F. Calidad de vida en niños y adolescentes con trastorno del espectro autista sin discapacidad intelectual. Rev Neurol 2016;62(Supl 1):S33-9.

16. García Coto MA. Autismo Infantil. Un estudio epidemiológico. [Tesis]. CONICET, 77, 1985.

17. Manzone L. Adaptación y validación del M-CHAT para población urbana argentina. Investigación e Innovación en Autismo. En Asociación Española de Profesionales del Autismo. Investigación e innovación en autismo: AETAPI, premios "Ángel Rivière". 5. ${ }^{\text {ta }}$ ed. Puerto Real: Asociación Española de Profesionales del Autismo; 2011:67-158.

18. Colantonio Llambías M, Salamanco G, Alfieri I, et al. Detección Precoz de Trastornos del Espectro Autista en niños de 18 a 30 meses en la consulta ambulatoria de un hospital público de C.A.B.A. $2^{\circ}$ Congreso Argentino de Discapacidad en Pediatría. 27-29 de sep. de 2012. Buenos Aires, Argentina; 2012.Págs.51-2.

19. Brown I, FriefeldS, Schiller C. Quality of life for persons with developmental disabilities: An annotated bibliography. The Centre for Health Promotion, University of Toronto, 2008. 
20. Gómez-Vela M, Verdugo MA. Cuestionario de evaluación de la Calidad de Vida de alumnos adolescentes (CCVA). Madrid: CEPE. 2009.

21. Verdugo MA, Gómez L, Arias B, et al. Escala San Martín: evaluación de la calidad de vida de personas con discapacidades significativas. Santander: Fundación Obra San Martín; 2014.

22. Council on Quality and Leadership. Cross-walk between the CMS HCBS Quality framework and CQL's "Quality Measures" 2005. [Acceso: 20 de septiembre de 2017]. Disponible en: http:/ / www.nasuad.org/sites/nasuad/ files/hcbs / files / 72/3580/Crosswalk05.pdf.

23. Cuesta Gómez JL. Trastornos del espectro autista y calidad de vida: Guía de indicadores para evaluar organizaciones y servicios. Madrid: La Muralla; 2009.

24. Frith U. Autismo. Madrid: Alianza; 1999.

25. Tamarit J. Autismo: modelos educativos para una vida de calidad. Rev Neurol 2005;40(Supl 1):181-6.

26. Martínez Martín MA, Cuesta Gómez JL. Todo sobre el autismo: Los trastornos del espectro autista. Guía completa basada en la ciencia y en la experiencia. Tarragona: Altaria; 2012.
27. Fuentes-Biggi J, Ferrari-Arroyo MJ, Boada-Muñoz L, et al. Guía de buena práctica para el tratamiento de los trastornos del espectro autista. Rev Neurol 2006;43:(7):425-38.

28. Ruiz Olabuénaga JI. Técnicas de triangulación y control de calidad en la investigación socioeducativa. Bilbao: Mensajero; 2003.

29. Cuesta Gómez JL. Aplicación de la técnica Delphi en el proceso de validación de un instrumento para la evaluación dela calidad de vida en centros para personas con Trastornos del Espectro Autista. Qurrículum 2013;26:135-60.

30. González Rey FL. Investigación cualitativa y subjetividad. Los procesos de construcción de la información. México: McGraw Hill; 2007.

31. Tójar Hurtado JC. Investigación cualitativa. Comprender y actuar. Madrid: La Muralla; 2006.

32. De la Herrán A. Investigar en educación: fundamentos, aplicación y nuevas perspectivas. Madrid: Dilex; 2005.

33. Landeta Rodríguez J. El método Delphi: una técnica de previsión de futuro. Barcelona: Ariel; 1999.

34. Schalock RL, Gardner JF, Bradley VJ. Quality of life for people with intellectual and other developmental disabilities. Washington: American Association on Intellectual and Developmental Disabilities; 2007. 
ANEXO 1.

Resumen del procedimiento de la aplicación de la técnica Delphi en la adaptación en Argentina de la guía de indicadores de calidad de vida para organizaciones que prestan apoyo a personas con trastornos del espectro autista (Cuesta, 2009)

\section{FASE PRELIMINAR}

- Definición de los criterios de selección del grupo de expertos

- Definición de las funciones

- Selección de los expertos

- Información y solicitud de participación en el grupo de expertos

- Preparación de la guía de indicadores por parte de los coordinadores
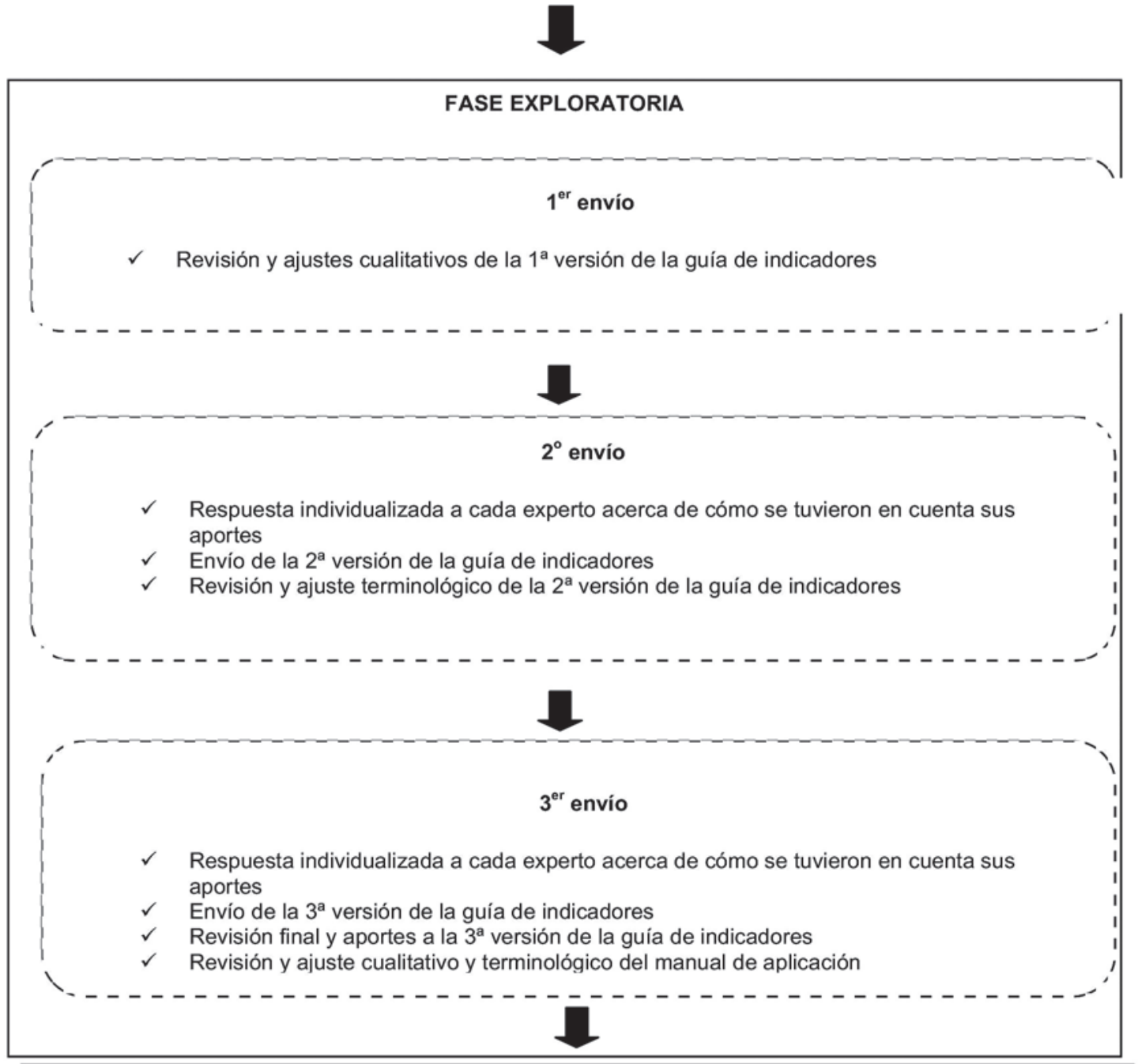

FASE FINAL

- $\quad$ Guía de indicadores definitiva (Anexo 2)

- Manual de aplicación 


\section{ANEXO 2 \\ GUÍA DE INDICADORES}

\section{CALIDAD REFERIDA A LA PERSONA}

\subsection{Calidad desde la perspectiva de la persona con TEA}

Bienestar físico

Indicador 1: Existen programas de atención sanitaria individualizados y actualizados.

Indicador 2: Se garantiza la correcta administración y seguimiento de los tratamientos farmacológicos.

Indicador 3: Se contempla la intervención individualizada en el ámbito del cuidado y autonomía personal. Indicador 4: Se desarrollan acciones referidas a la seguridad e higiene en los diferentes ámbitos.

Indicador 5: Se contemplan medidas preventivas individualizadas para mantener una salud adecuada.

Bienestar emocional

Indicador 6: El ambiente promueve un estado emocional positivo en las personas con TEA.

Indicador 7: Se promueve la máxima estabilidad emocional en la vida de las personas con TEA.

Indicador 8: Se desarrollan programas individualizados de intervención conductual positiva.

Indicador 9: La persona con TEA y/o su tutor participan en la planificación, ejecución y evaluación de su plan de elaboración individual.

Indicador 10: Las personas con TEA cuentan con apoyos personales individualizados.

Bienestar material

Indicador 11: Se respeta la intimidad.

Indicador 12: Se promueven y respetan las pertenencias.

Relaciones interpersonales

Indicador 13: Se promueven las relaciones sociales significativas.

Desarrollo personal

Indicador 14: Se promueve el desarrollo de las capacidades e intereses individuales.

Indicador 15: Se promueve el avance y el desarrollo continuo de la persona. Derechos

Indicador 16: Se garantiza el respeto a la identidad y dignidad de la persona.

Indicador 17: Se garantiza la integridad física.

Autodeterminación

Indicador 18: Las personas reciben formación variada y adaptada, previa a la emisión de conductas de autodeterminación.

Indicador 19: Las personas participan en la planificación y ejercen un control sobre su vida.

Inclusión social

Indicador 20: Se promueve la inclusión social de las personas con TEA.

\subsection{Calidad desde la perspectiva de las familias}

Indicador 21: Las acciones iniciadas con la persona con TEA tienen en cuenta las expectativas de la familia.

Indicador 22: Se facilita la implicación de las familias en la organización.

Indicador 23: Se favorece un aumento del nivel de satisfacción en las familias.

\subsection{Calidad desde la perspectiva de los profesionales}

Indicador 24: Se conocen, evalúan y se tienen en cuenta las propuestas e iniciativas provenientes de los profesionales.

Indicador 25: Las responsabilidades de los profesionales son coherentes con sus funciones.

Indicador 26: Se promueve la participación y el trabajo en equipo.

Indicador 27: Se favorece un aumento del nivel de satisfacción en los profesionales.

Indicador 28: Los profesionales están implicados en la organización.

\section{IDENTIFICACIÓN DE LAS NECESIDADES Y PREFERENCIAS/ELABORACIÓN Y SEGUIMIENTO DE LOS PLANES DE DESARROLLO PERSONAL}

\subsection{Programación}

Indicador 29: Se evalúan las necesidades, aspiraciones y expectativas de las personas con TEA en los distintos ámbitos de intervención. 
Indicador 30: Se elaboran planes de intervención adaptados a las necesidades específicas a lo largo de toda la vida.

Indicador 31: La estructura de la programación general de la organización se adapta a las características de las personas con TEA.

Indicador 32: Se adecua el proceso de elaboración de los planes de desarrollo personal a las características de las personas con TEA.

\subsection{Planificación de apoyos}

Indicador 33: Existe un proceso de planificación y utilización de apoyos que responde a las características de cada persona.

Indicador 34: Los criterios metodológicos se adaptan a las necesidades y capacidades de la persona con TEA.

Indicador 35: Los planes de desarrollo personal se adaptan a la persona.

\subsection{Plan de seguimiento y evaluación}

Indicador 36: Existe un seguimiento y evaluación continua de cada plan de desarrollo personal.

\section{FORMACIÓN DE LOS PROFESIONALES}

\subsection{Conocimiento del autismo}

Indicador 37: Se asegura una formación inicial a los nuevos profesionales.

Indicador 38: La formación incluye aspectos técnicos, organizacionales y valores de la organización.

Indicador 39: Cada profesional recibe una formación específica sobre su puesto de trabajo.

Indicador 40: Se fomenta la formación continua, la actualización y el desarrollo profesional.

Indicador 41: La entidad cuenta con recursos propios que favorecen la formación, actualización y desarrollo profesional.

\subsection{Conocimiento y adaptación a la persona con TEA: planificación centrada en la persona (PCP)}

Indicador 42: El accionar de cada profesional tiene como referencia los principios de la PCP.

Indicador 43: Existe una información individualizada de cada persona con TEA.

Indicador 44: Se conoce en profundidad y de forma integral a la persona con TEA.

Indicador 45: La intervención es adaptada a cada plan de desarrollo personal (características, necesidades, aspiraciones y expectativas, preferencias de la persona, etc.).

Se tienen en cuenta las necesidades individuales de apoyo específico y/o especializado.

\subsection{Actitudes y valores}

Indicador 46: La práctica y actitudes profesionales tienen como referente la misión y los valores de la organización.

\subsection{Participación en investigaciones sobre trastorno del espectro autista}

Indicador 47: La organización fomenta la ampliación del conocimiento a través de la participación activa en investigaciones sobre TEA.

\section{ESTRUCTURA Y ORGANIZACIÓN}

\subsection{Grupos de compañeros}

Indicador 48: La conformación de los grupos de compañeros se adapta a las necesidades de las personas con TEA.

Indicador 49: Los profesionales son una referencia clara para las personas con TEA.

\subsection{Organización de la actividad}

Indicador 50: Existe una organización de las tareas y actividades.

Indicador 51: Las personas con TEA tienen asignadas responsabilidades y participan activamente en la organización.

Indicador 52: Se dispone de apoyo y seguimiento técnico integrado en el equipo profesional. 


\subsection{Horario}

Indicador 53: El horario y ritmo de trabajo de las personas con TEA se adapta a sus necesidades.

Indicador 54: El horario y distribución de tiempos de los profesionales se adecua a las necesidades de las personas con TEA.

\subsection{Comunicación/coordinación}

Indicador 55: Se facilita la comunicación entre todas las personas vinculadas a la organización.

Indicador 56: Se planifican y promueven tiempos y espacios para la coordinación.

Indicador 57: Existe coordinación con otros programas y servicios relacionados con la persona con TEA. Indicador 58: Se facilita la comunicación a las personas con TEA.

\subsection{Evaluación sistemática del servicio y/o la organización}

Indicador 59: Se realiza una evaluación interna de la organización.

Indicador 60: La mejora de la organización contempla una evaluación externa.

\subsection{Liderazgo}

Indicador 61: La dirección de la organización impulsa la mejora continua.

\section{RECURSOS Y SERVICIOS}

Indicador 62: Se utilizan los recursos humanos.

Indicador 63: Existe una adecuada organización del trabajo de los profesionales.

Existe una estructuración clara de los tiempos, actividades y grupos asignados a cada profesional a lo largo de toda la jornada.

Indicador 64: Se utilizan los recursos materiales. Los recursos están a disposición de todos los profesionales.

Indicador 65: El entorno físico favorece la participación, accesibilidad y la autonomía de las personas con TEA.

\section{RELACIÓN CON LA COMUNIDAD/PROYECCIÓN SOCIAL}

Indicador 66: Existen alianzas de colaboración con otras entidades, pertenezcan o no al sector de la discapacidad.

Indicador 67: La organización asume un compromiso de responsabilidad social.

Existe un plan de comunicación externa para divulgar y ofrecer información sobre autismo: folletos de divulgación, página web, publicaciones, videos, aparición en medios de comunicación, etc. 